\title{
FREE ENERGY AND ENTROPY FOR AN IMPURITY IN A PERIODIC BACKGROUND IN ONE DIMENSION
}

\author{
M. Bordag ${ }^{1 *}$, I.G. Pirozhenko ${ }^{1,2}$, \\ ${ }^{1}$ Institute for Theoretical Physics, Universität Leipzig, Brüderstr. 16, 04081 Leipzig, Germany \\ ${ }^{2}$ Bogoljubov Institute for Theoretical Physics, Joint Institute for Nuclear Research, JINR Dubna State \\ University, 141980 Dubna, Moscow region, Russia \\ *e-mail: bordag@uni-leipzig.de
}

In this paper, we continue our study of periodic lattices formed by delta functions and their derivatives in $(1+1)$ dimensions. Such systems are interesting as allowing for investigation of complicated problems in quite simple terms. Specifically, we consider the free energy of a single impurity in the background of such lattice. After developing the necessary technical tools, we consider as an example a specific case and found that the free energy shows a non monotone behavior as function of the temperature.

Keywords: vacuum energy, Casimir effect, periodic lattice, impurity, free energy.

Received 12.10.2020; Received in revised form 15.11.2020; Accepted 01.12.2020

\section{Introduction}

One-dimensional systems are an excellent laboratory for studying quantum effects.On the one hand, sometimes it is worth reducing the problem to one spatial dimension in order to obtain explicit and instructive formulas. On the other hand, one-dimensional systems have their own unique properties [1], for example, the standard description of electron gas in a solid in terms of quasiparticles breaks down for $D=1$, where only collective excitations may exist. Consequently, the electron transport differs from that in 2- and 3-dimensional materials. Nanowires and carbon nanotubes should be mentioned as such one-dimensional and quasi-one-dimensional systems that have acquired practical importance in the last decades. Another example is the 1D quantum liquid, which cannot be described by Fermi-liquid theory and at low energies has a gapless excitation spectrum with linear dispersion [2].

A one-dimensional system may be manufactured by trapping ultra cold atoms in an optical lattice made by interfering lasers. It is modeled by periodic potentials with a point support. The simplest one-dimensional model is the "Dirac comb" with a Dirac delta functions in each cell, being a special case of a Kronig-Penney (KP) potential [3]. Such potentials are also known as 'zero-range potentials' and appear in various contexts [4], for example, in connection with the Bose-Einstein condensation [5-7] or quantum liquids with impurities [2].

A straightforward generalization of the KP model is to include a derivative of a delta function $\left(\delta^{\prime}\right.$ - potential), see equation 3 below. The most general case of zero range potentials can be found in [8] and, with applications to metamaterials, in [9]. For the discussion of selfadjointness for general operators with point support see [10].

The effect of impurities on the band structure in crystals is of actual interest from both theoretical and experimental points of view. It has been suggested in [11] that in the neighbourhood of a defect there is a bend in the conduction band. In [12] this idea was explored using one-dimensional KP model with an impurity. The influence of impurities on the transmission coefficient and their relationship with localization was analyzed in [13]. In [14] the KP model with arbitrary positions and forces of scattering centers was studied. Its particular case is an almost periodic model with one or a few impurities. Here an interesting development is the KP model with modulated scatterer heights, which can be applied in solid state and optical lattices.

In this paper, a one-dimensional scalar field is considered in the background formed by a lattice of generalized delta functions with a point impurity. We study the influence of the impurity on the free energy and the entropy of the systems. This is an addition to the research of 
the vacuum energy, free energy and the entropy started in articles [15-17] for periodic potentials of generalized delta functions with and without impurities. In the weak coupling regime the fluctuation forces acting on an impurity were analyzed in the very recent paper [18].

The outline of the paper is as follows. Section 2 introduces general formulas for the free energy and the entropy in terms of a mode generating function. Section 3 is devoted to scattering on a $1 \mathrm{D}$ lattice of generalized $\delta$-functions. Here we derive the transfer matrix and the dispersion relation. In Section 4 the translational invariance of the potential is broken by one impurity which is a delta function with different parameters located inside one of the lattice cells. In section 5 the integral representation for the free energy of the impurity is obtained. Finally, we present and discuss some numerical results for the free energy and the entropy for an impurity in one dimensional periodic background.

We use the system of units $\bar{h}=c=1$.

\section{The free energy}

We use the standard expression

$$
\begin{aligned}
& \mathcal{F}=\sum_{j}\left[\frac{\omega_{j}^{1-2 s}}{2}+T \ln \left(1-e^{-\beta \omega_{j}}\right)\right], \\
& \equiv E_{0}(s)+\Delta_{T} \mathcal{F},
\end{aligned}
$$

for the free energy of a system with discrete eigenfrequencies $\omega_{j}$. Here $\beta=1 / T$ is the inverse temperature and the sum extends over the whole spectrum. Since this sum includes, with the first term in the square bracket, the vacuum energy, $E_{0}(s)$, it contains an ultraviolet divergence. For this reason we introduced the zeta functional regularization by raising the frequency to a power with sufficiently large regularization parameter $s$. After normalization one must take $s=0$. The second term in the square bracket is the temperature dependent part of the free energy, $\Delta_{T} \mathcal{F}$, and it is convergent.

It is convenient to transform the sum into an integration. Using a contour $\gamma$ which encircles all eigenfrequencies $\omega_{j}$, we get

$$
\mathcal{F}=\int_{\gamma} \frac{d \omega}{2 \pi i}\left[\frac{\omega^{1-2 s}}{2}+T \ln \left(1-e^{-\beta \omega}\right)\right] \frac{\partial}{\partial \omega} \ln \Delta(\omega)
$$

where $\Delta(\omega)$ is the mode generating function, i.e., the discrete eigenfrequencies $\omega_{j}$ are the solutions of the equation $\Delta(\omega)=0$. Tightening the contour around the real $\omega$-axis, the expressions is transformed into

$$
\mathcal{F}=\int_{0}^{\infty} \frac{d \omega}{\pi}\left[\frac{\omega^{1-2 s}}{2}+T \ln \left(1-e^{-\beta \omega}\right)\right] \frac{\partial}{\partial \omega} \delta(\omega)
$$

with the 'phase'

$$
\delta(\omega)=\frac{1}{2 i} \ln \frac{\Delta(\omega-i 0)}{\Delta(\omega+i 0)} .
$$

The factor $\Delta(\omega+i 0)$ results from the part of the integration path above the axis and $\Delta(\omega-i 0)$ from the part below. It must be mentioned that eq. 3 is a generalization of the 'remarkable formula' derived and discussed in [19], or later, in [20] and [21] for systems with dissipation. 
From this representation it is also clear that $\frac{\partial}{\partial \omega} \delta(\omega)$ can be interpreted as density of states.

Another change in the representation can be done by integrating by parts,

$$
\mathcal{F}=-\int_{0}^{\infty} \frac{d \omega}{\pi}\left[\frac{(1-2 s) \omega^{-2 s}}{2}+\frac{1}{e^{\beta \omega}-1}\right] \delta(\omega),
$$

where the second term in the square bracket is the Boltzmann factor.

We mention also the relation to the Matsubara representation. By splitting the logarithm in 4 into a sum of two, and turning the integration path in $\ln \Delta(\omega+i 0)$ towards the imaginary axis, which is equivalent to a Wick rotation, and turning the path in the other term towards the negative imaginary axis, we arrive at

$$
\mathcal{F}=(1-2 s) \cos (\pi s) T \sum_{\ell \geq 0}^{\prime} \xi_{\ell}^{-2 s} \ln \Delta\left(i \xi_{\ell}\right),
$$

where the prime indicates that the term with $\ell=0$ enters with the weight $\frac{1}{2}$ and $\xi_{\ell}=2 \pi T \ell$ are the Matsubara frequencies. These come in from the poles the Boltzmann factor has on the imaginary frequency axis.

The corresponding expression for the entropy

$$
S=-\frac{\partial}{\partial T} \mathcal{F}
$$

reads

$$
S=\int_{0}^{\infty} \frac{d \omega}{\pi} \frac{-\beta^{2} \omega e^{\beta \omega}}{\left(e^{\beta \omega}-1\right)^{2}} \delta(\omega) .
$$

It is free from ultraviolet divergences.

\section{The lattice}

We consider a scalar field obeying the equation

$$
\left(-\omega^{2}+\frac{\partial}{\partial x}+V(x)\right) \phi(x)=0
$$

with a potential resulting from a lattice of delta functions and their derivatives,

$$
V_{\delta}(x)=\sum_{n}\left(\alpha \delta(x-a n)+2 \beta \delta^{\prime}(x-a n)\right) .
$$

This potential is realized by matching conditions

$$
\left(\begin{array}{c}
\phi(a n+0) \\
\phi^{\prime}(a n+0)
\end{array}\right)=M\left(\begin{array}{c}
\phi(a n-0) \\
\phi^{\prime}(a n-0)
\end{array}\right)
$$

the field must obey. The matrix $M$ depends on the parameters of the potential,

$$
\boldsymbol{M}=\left(\begin{array}{cc}
\frac{1+\beta}{1-\beta}, & 0 \\
\frac{\alpha}{1-\beta^{2}}, & \frac{1-\beta}{1+\beta}
\end{array}\right)
$$


This model is a modification of the well known Kronig-Penney model [3]. For $\beta=0$ the potential consists of simple delta functions and is also known as 'Dirac comb'. Generalizations can be found in [22].

For the solutions we make the ansatz

$$
\phi(x)=\sum_{n=-\infty}^{\infty} \phi_{n}(x) \Theta_{n}
$$

with the characteristic function of the cells,

$$
\Theta_{n}= \begin{cases}1 & \text { if }(n-1) a \leq x \leq a n, \\ 0 & \text { otherwise }\end{cases}
$$

For the function in the cells we take linear combinations of plane waves

$$
\phi_{n}(x)=A_{n} e^{i \omega(x-a n)}+B_{n} e^{-i \omega(x-a n)} .
$$

The coefficients $A_{n}$ and $B_{n}$ are related by the matching conditions 11,

$$
\left(\begin{array}{c}
A_{n+1} \\
B_{n+1}
\end{array}\right)=\boldsymbol{T}\left(\begin{array}{c}
A_{n} \\
B_{n}
\end{array}\right), \quad(n=-\infty, \ldots, \infty),
$$

with the transfer matrix

$$
\boldsymbol{T}=\boldsymbol{Q}^{-1} \boldsymbol{K}^{-1} \boldsymbol{M} \boldsymbol{K}, \quad \boldsymbol{Q}=\left(\begin{array}{lll}
e^{-i \omega a} & , & 0 \\
0 & , & e^{i \omega a}
\end{array}\right), \quad \boldsymbol{K}=\left(\begin{array}{lll}
1 & , & 1 \\
i \omega & , & -i \omega
\end{array}\right) .
$$

Inserting 12 one arrives at

$$
\boldsymbol{T}=\left(\begin{array}{lll}
w & , & z \\
z^{*} & , & w^{*}
\end{array}\right)
$$

with

$$
w=\frac{1+\beta^{2}-\frac{i \alpha}{2 \omega}}{1-\beta^{2}} e^{i \omega a}, \quad z=\frac{2 \beta-\frac{i \alpha}{2 \omega}}{1-\beta^{2}} e^{i \omega a} .
$$

For real $\omega$, of course, the relations

$$
\operatorname{det} \boldsymbol{T}=1, \quad \text { or equivalently, }|w|^{2}-|z|^{2}=1,
$$

hold. The periodicity of the lattice results in the Bloch condition,

$$
\phi(x+a)=e^{i q a} \phi(x),
$$

where $q$ is the quasi momentum. This property provides a second relation, beyond 16 between the coefficients,

$$
\left(\begin{array}{c}
A_{n+1} \\
B_{n+1}
\end{array}\right)=e^{i q a}\left(\begin{array}{c}
A_{n} \\
B_{n}
\end{array}\right)
$$


Combining 16 and 22 we get with

$$
\left(\boldsymbol{T}-e^{i q a}\right)\left(\begin{array}{c}
A_{n} \\
B_{n}
\end{array}\right)=0 .
$$

an equation for the coefficients. Since this is a homogeneous system, the determinant must vanish,

$$
\operatorname{det}\left(\boldsymbol{T}-e^{i q a}\right)=0 .
$$

Using 18 and 19, we get the dispersion relation

$$
\cos (q a)=h(\omega)
$$

with

$$
h(\omega) \equiv \frac{w+w^{*}}{2}=\frac{\left(1+\beta^{2}\right) \cos (\omega a)+\frac{\alpha}{2 \omega} \sin (\omega a)}{1-\beta^{2}} .
$$

This equation determines the energy bands of the model. In order to have normalizable wave functions, because of the translation property 21 , we need to have real quasi momentum $q$. This is the case if the condition

$$
-1 \leq h(\omega) \leq 1
$$

holds.

The dispersion relation 25 has two solutions. We define

$$
q=\frac{1}{a} \arccos h(\omega)
$$

on the main branch. The second solution has $-q$. Accordingly, we have two solutions 13 ,

$$
\phi^{ \pm}(x)=\sum_{n=-\infty}^{\infty} \phi_{n}^{ \pm}(x) \Theta_{n}, \quad \phi_{n}^{ \pm}(x)=A_{n}^{ \pm} e^{i \omega(x-a n)}+B_{n}^{ \pm} e^{-i \omega(x-a n)}
$$

which are defined by

$$
\phi_{n+1}^{ \pm}(x)=e^{ \pm i q a} \phi_{n}^{ \pm}(x) .
$$

Solving the equation 23 , we get for the coefficients

$$
\left(\begin{array}{c}
A_{1}^{+} \\
B_{1}^{+}
\end{array}\right)=\left(\begin{array}{c}
w^{*}-e^{i q a} \\
-z^{*}
\end{array}\right),\left(\begin{array}{c}
A_{1}^{-} \\
B_{1}^{-}
\end{array}\right)=\left(\begin{array}{c}
-z \\
w-e^{-i q a}
\end{array}\right),
$$

which completes the description of the solutions.

It should be mentioned that the above formulas are not new. Similar ones can be found in literature. We take reference for instance to [23], [15], [24] and [25]. Also we mention a slightly different derivation of the formulas 31 for the coefficients in the wave function in [26]. 


\section{The impurity}

As impurity we take a potential like 10 with different parameters,

$$
V_{\text {imp. }}=\tilde{\alpha} \delta\left(x-x_{0}\right)+2 \tilde{\beta} \delta^{\prime}\left(x-x_{0}\right),
$$

located at $x_{0}$ and we assume $0 \leq x_{0} \leq a$. The impurity beaks the translational invariance of the lattice. We make the ansatz

$$
\phi_{\text {imp. }}(x)=\left(\mu_{L} \phi^{+}(x)+\nu_{L} \phi^{-}(x)\right) \Theta\left(-x+x_{0}\right)+\left(\mu_{R} \phi^{+}(x)+\nu_{R} \phi^{-}(x)\right) \Theta\left(x-x_{0}\right)
$$

for its wave function. The potential is realized by the matching conditions

$$
\left(\begin{array}{c}
\phi_{\text {imp. }}\left(x_{0}+0\right) \\
\phi_{\text {imp. }}^{\prime}\left(x_{0}+0\right)
\end{array}\right)=\widetilde{\boldsymbol{M}}\left(\begin{array}{c}
\phi_{\text {imp. }}\left(x_{0}-0\right) \\
\phi_{\text {imp. }}^{\prime}\left(x_{0}-0\right)
\end{array}\right)
$$

with

$$
\widetilde{\boldsymbol{M}}=\left(\begin{array}{cc}
\frac{1+\tilde{\beta}}{1-\tilde{\beta}}, & 0 \\
\frac{\tilde{\alpha}}{1-\tilde{\beta}^{2}}, & \frac{1-\tilde{\beta}}{1+\tilde{\beta}}
\end{array}\right)
$$

Inserting here the ansatz 33 we arrive at

$$
\left(\begin{array}{c}
\mu_{R} \\
\nu_{R}
\end{array}\right)=\widetilde{\boldsymbol{T}}\left(\begin{array}{c}
\mu_{L} \\
\nu_{L}
\end{array}\right)
$$

with

$$
\widetilde{\boldsymbol{T}}=\widetilde{\boldsymbol{K}}^{-1} \widetilde{\boldsymbol{M}} \widetilde{\boldsymbol{K}}, \quad \widetilde{\boldsymbol{K}}=\left(\begin{array}{c}
\phi^{+}\left(x_{0}\right), \phi^{-}\left(x_{0}\right) \\
\phi^{\prime+}\left(x_{0}\right), \phi^{\prime-}\left(x_{0}\right)
\end{array}\right)
$$

The impurity wave function $\phi_{\text {imp. }}(x), 33$, has been investigated in detail in [26]. For instance, the scattering problem was considered and the transmission coefficient was calculated. Also, the bound state solutions were found. These exist for negative $\tilde{\alpha}$. For instance, it was shown that there is a region with $\alpha>0$, where a bound state has positive energy. This is important for the stability of the vacuum energy since otherwise it would have an imaginary part.

\section{The free energy of the impurity}

The vacuum energy, $E_{0}$, of the lattice itself was calculated [15] and, by another method, in [26], where also the vacuum energy of the impurity was calculated. The free energy of the comb was calculated in [17]. In this section we calculate the temperature dependent part of the free energy of the impurity. For this calculation we adopt a modified scheme which appears to be easier.

In order to take advantage of the formulas presented in Sect. 2, we put the system into a finite box by imposing Dirichlet boundary conditions at the ends,

$$
\phi\left(L_{1}\right)=0, \quad \phi\left(L_{2}\right)=0,
$$


with $L_{1}<x_{0}<L_{2}$. The boundary points are parameterized as follows,

$$
L_{1,2}=a m_{1,2}+\Delta L_{1,2}, \quad m_{1,2}=\left[L_{1,2}\right]+1, \quad(i=1,2) .
$$

To remove the box, we will tend $m_{1} \rightarrow \infty$ and $m_{2} \rightarrow-\infty$, whereby $\Delta L_{1}$ and $\Delta L_{2}$ remain in the basic interval, $0 \leq \Delta L_{1,2} \leq a$. This way it is guaranteed that the position $x_{0}$ of the impurity is always in-between $L_{1}$ and $L_{2}$.

Basically, we need the box to get discrete frequencies $\omega_{j}$ and the corresponding mode generating function $\Delta(\omega)$. To get it, we insert the ansatz 33 for the impurity wave function into 38 ,

$$
\begin{aligned}
\left(\mu_{L} \phi^{+}\left(L_{1}\right)+\nu_{L} \phi^{-}\left(L_{1}\right)\right) & =0, \\
\left(\mu_{R} \phi^{+}\left(L_{2}\right)+\nu_{R} \phi^{-}\left(L_{2}\right)\right) & =0 .
\end{aligned}
$$

We substitute $\mu_{R}$ and $\nu_{R}$ using 36 and get a system of equations for $\mu_{L}$ and $\nu_{L}$. This is a homogeneous system and its determinant must vanish,

$$
\begin{aligned}
\Delta(\omega) & \equiv \tilde{T}_{11} \phi^{+}\left(L_{2}\right) \phi^{-}\left(L_{1}\right)-\tilde{T}_{22} \phi^{-}\left(L_{2}\right) \phi^{+}\left(L_{1}\right)+\tilde{T}_{21} \phi^{-}\left(L_{2}\right) \phi^{-}\left(L_{1}\right)-\tilde{T}_{12} \phi^{+}\left(L_{2}\right) \phi^{+}\left(L_{1}\right), \\
& =0 .
\end{aligned}
$$

The solutions of this equation are just the frequencies $\omega_{j}$.

We consider the temperature dependent part of the free energy in 1 and use 3 with 4 to get

$$
\Delta_{T} \mathcal{F}=\int_{0}^{\infty} \frac{d \omega}{\pi} T \ln \left(1-e^{-\beta \omega}\right) \frac{\partial}{\partial \omega} \delta(\omega) .
$$

Now we want to remove the box. For this, as preparation, we use 30 to rewrite 41 ,

$$
\begin{aligned}
\Delta(\omega) \equiv & \tilde{T}_{11} e^{i q a\left(m_{2}-m_{1}\right)} \phi^{+}\left(\Delta L_{2}\right) \phi^{-}\left(\Delta L_{1}\right)-\tilde{T}_{22} e^{i q a\left(m_{1}-m_{2}\right)} \phi^{-}\left(\Delta L_{2}\right) \phi^{+}\left(\Delta L_{1}\right) \\
& +\tilde{T}_{21} e^{i q a\left(-m_{2}-m_{1}\right)} \phi^{-}\left(\Delta L_{2}\right) \phi^{-}\left(\Delta L_{1}\right)-\tilde{T}_{12} e^{i q a\left(m_{2}+m_{1}\right)} \phi^{+}\left(\Delta L_{2}\right) \phi^{+}\left(\Delta L_{1}\right) .
\end{aligned}
$$

As announced, we tend $m_{2} \rightarrow \infty$ and $m_{1} \rightarrow-\infty$ and account for the imaginary part of $\omega$ in $\delta$, 4. The imaginary part of q, 28 follows that of $\omega$. In $\Delta(\omega+i 0)$, the second term in 41 dominates and in $\Delta(\omega-i 0)$, the first term in 41 dominates. The other are exponentially smaller and we drop them. This way, we get

$$
\delta(\omega) \simeq q a\left(m_{2}-m_{1}\right)+\frac{1}{2 i} \ln \frac{\phi^{+}\left(\Delta L_{2}\right) \phi^{-}\left(\Delta L_{1}\right)}{\phi^{-}\left(\Delta L_{2}\right) \phi^{+}\left(\Delta L_{1}\right)}+\frac{1}{2 i} \ln \frac{\tilde{T}_{11}}{\tilde{T}_{22}} .
$$

We rewrite this expression in the form

$$
\delta(\omega)=\delta_{0}+\delta_{1}+\delta_{2}
$$


with

$$
\begin{aligned}
& \delta_{0}=q\left(L_{2}-L_{1}\right), \quad \delta_{1}=q\left(\Delta L_{1}-\Delta L_{2}\right)+\frac{1}{2 i} \ln \frac{\phi^{+}\left(\Delta L_{2}\right) \phi^{-}\left(\Delta L_{1}\right)}{\phi^{-}\left(\Delta L_{2}\right) \phi^{+}\left(\Delta L_{1}\right)}, \\
& \delta_{2}=\frac{1}{2 i} \ln \frac{\tilde{T}_{11}}{\tilde{T}_{22}} .
\end{aligned}
$$

Here, $\delta_{0}$ is the contribution from the empty lattice, $\delta_{1}$ is reminiscent of the position of the box relative to the lattice, and $\delta_{3}$ is the contribution from the impurity.

The contributions from the lattice were considered in [15] and [17] and we focus on the third part, i.e, on the free energy of the impurity,

$$
\Delta_{T} \mathcal{F}=\int_{0}^{\infty} \frac{d \omega}{\pi} T \ln \left(1-e^{-\beta \omega}\right) \frac{\partial}{\partial \omega} \delta_{2}(\omega)
$$

Finally, we integrate by parts like in 5 ,

$$
\Delta_{T} \mathcal{F}=-\int_{0}^{\infty} \frac{d \omega}{\pi} \frac{1}{e^{\beta \omega}-1} \frac{1}{2 i} \ln \frac{\tilde{T}_{11}}{\tilde{T}_{22}}
$$

where 46 was used. This representation is convenient for numerical evaluation. An example is shown in Fig. 1.

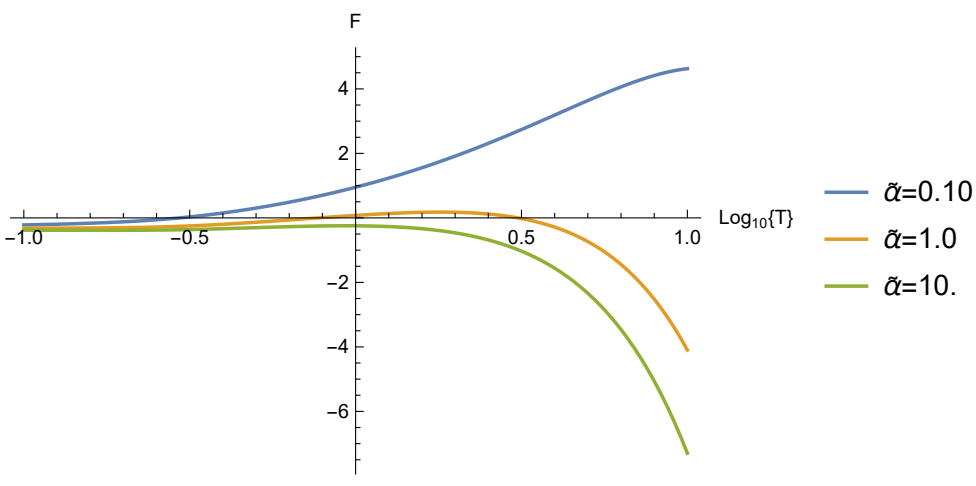

Fig. 1. The free energy of a single impurity for several values of its strength $\tilde{\alpha}$ in the background of the lattice as function of the temperature $T$. The parameters of the lattice are $\beta=0, \alpha=0.3$ and $x_{3}=0.1$.

\section{Conclusions}

In the forgoing sections we presented the theory of how to calculate the free energy of an impurity in the background of a periodic lattice. Both, lattice and impurity, were modeled by delta functions, which allows for quite explicit formulas. For this model, the free energy of the pure lattice was investigated in an earlier paper [17] and showed a very monotone behavior, see Fig. 4 in the mentioned paper. The contribution from the impurity, calculated in Sect. 5, shows a non monotone behavior. This is interesting as it gives a hint on possible negative entropy in the considered system. Examples for that were found earlier, see for instance, Fig. 11 in [17].

From a technical point of view, we would like to mention that the derivation of the free energy, found in the present paper, is considerable easier as compared to [17]. Especially, with eq. 46 we got a clear distinction between the individual contributions. This approach will allow for more detailed investigations of the thermodynamics of impurities in future work. 


\section{References}

1. Giamarchi, T. One-dimensional physics in the 21st century / Thierry Giamarchi // Comptes Rendus Physique, Condensed matter physics in the 21st century: The legacy of Jacques Friedel. - 2016. - Vol. 17(3). - P. 322-331.

2. Recati, A. Casimir forces between defects in one-dimensional quantum liquids /, A Recati, JN Fuchs, CS Peca, and W Zwerger// Phys. Rev. A - 2005. - Vol. 72(2). - 023616.

3. Kronig, R. de L. Quantum Mechanics of Electrons in Crystal Lattices / R. de L. Kronig and W. G. Penney // Proc. R. Soc. A - 1931. - Vol. 130. - P. 499.

4. Bordag, M. Dirac Lattices, Zero-Range Potentials and Self Adjoint Extension / M. Bordag and J.M. Muñoz Castañeda // Phys. Rev. D - 2015. - Vol. 91. - 065027.

5. Barragan, V. E. Bose gas in disordered, finite-layered systems /, V. E. Barragan, M. Fortes, M. A. Solis, and P. Salas // International Journal Of Modern Physics B - 2016. - 30.

6. Bordag, M. On Bose-Einstein condensation in one-dimensional lattices of delta functions /, M. Bordag // Mod. Phys. Lett. - 2020 - Vol. A35(03). - 2040005.

7. Bordag, M. Conditions for Bose-Einstein condensation in periodic background /, M. Bordag // J. Phys. - 2020. - Vol. A53(1). -015003.

8. Garcia, D. Casimir effect and global theory of boundary conditions /, M. Asorey, D. Garcia Alvarez, and J. M. Munoz-Castaneda // J. Phys. A: Math. Gen. - 2006. - Vol. 39. - P. 6127 $-6136$.

9. Nieto, L. M. Towards modelling QFT in real metamaterials: Singular potentials and selfadjoint extensions / L. M. Nieto, M Gadella, J. Mateos Guilarte, J. M. Muñoz-Castañeda, and C. Romaniega // Journal of Physics: Conference Series - 2017. - Vol. 839. - 012007.

10. Kurasov, P. Distribution theory for discontinuous test functions and differential operators with generalized coefficients / P. Kurasov // Journal of Mathematical Analysis and Applications - 1996. - Vol. 201(1). - P. 297 - 323.

11. Baran, A. Binding energies of Eu2+ and Eu3+ ions in beta-Ca2SiO4 doped with europium/, Anna Baran, Justyna Barzowska, Marek Grinberg, Sebastian Mahlik, Karol Szczodrowski, and Yuriy Zorenko // Optical Materials - 2013. - Vol. 35(12). - P. 2107 - 2114.

12. Mihokova, E. Energy bands and gaps near an impurity /, E. Mihokova and L. S. Schulman // Phys. Lett. A - 2016. - Vol. 380(41). - P. 3430 - 3433.

13. Dharani, M. Band structures in transmission coefficients generated by dirac comb potentials / M. Dharani and C.S. Shastry // Physica B: Condensed Matter - 2016. - Vol. 500. P. $66-76$.

14. Reshodko, I. Topological states in the Kronig-Penney model with arbitrary scattering potentials / Irina Reshodko, Albert Benseny, Judit Romhányi, and Thomas Busch // New Journal of Physics - 2019. - Vol. 21(1). - 013010.

15. Bordag, M. Vacuum energy for generalised Dirac combs at $T=0 /$ Michael Bordag, Jose M. Muñoz Castañeda, and Lucia Santamaría-Sanz // Front. Phys. - 2019. - Vol. 7. 38.

16. Muñoz Castañeda, J. M. Revisiting the Casimir Energy with General Boundary Conditions and applications in 1D Crystals / J. M. Muñoz Castañeda, M. Bordag, and L. SantamaríaSanz // Mod. Phys. Lett. - 2020. - Vol. A35(03). - 2040018.

17. Bordag, M. Free energy and entropy for finite temperature quantum field theory under the influence of periodic backgrounds / M. Bordag, J. M. Munoz-Castaneda, and L. SantamaríaSanz // Eur. Phys. J. C - 2020. - Vol. 80(3).

18. Reichert, B. Fluctuation-induced potential for an impurity in a semi-infinite one- 
dimensional Bose gas / Benjamin Reichert, Aleksandra Petkovic, and Zoran Ristivojevic // Phys. Rev. B - 2019. - Vol. 100(23).

19. Ford, G. W. Quantum Oscillator in a Blackbody Radiation Field / G. W. Ford, J. T. Lewis, and R. F. O'Connell // Phys. Rev. Lett. - 1985. - Vol. 55. - P. 2273 - 2276.

20. Intravaia, F. Casimir Effect as a Sum over Modes in Dissipative Systems / Francesco Intravaia and Ryan Behunin // Phys. Rev. A - 2012. - Vol. 86.

21. Bordag, M. Vacuum and thermal energies for two oscillators interacting through a field/ M. Bordag // Theor. Mat. Phys. - 2018. - Vol. 195. - 834.

22. Asorey, M. Global Theory of Quantum Boundary Conditions and Topology Change / M. Asorey, A. Ibort, and G. Marmo // Int. J. Mod. Phys. A - 2005. - Vol. 20. - P. 1001 1025.

23. Gadella, M. Bound states and scattering coefficients of the $-a \delta(x)+b \delta^{\prime}(x)$ potential / M. Gadella, J. Negro, and L.M. Nieto // Phys. Lett. A - 2009. - Vol. 373(15). - P. 1310 1313.

24. Botman, S. Bloch wave scattering on pseudopotential impurity in 1D Dirac comb model/ Stepan Botman and Sergey Leble // 2015. - arXiv: 1511.04758.

25. Botman, S. Bloch wave-ZRP scattering as a key element of solid state physics computation: 1D example / Stepan Botman and Sergey Leble // TASK Quarterly - 2016. - Vol. 20197. - arXiv: 1511.04758 .

26. Bordag, M. Casimir effect for impurity in periodic background in one dimension / M Bordag // J. Phys. A: Math. Gen. - 2020. - Vol. 53(32) - 325401. 